DOI:10.5216/cab.v15i1.23327

\title{
VALIDAÇÃO DE UM PROGRAMA DE PRODUÇÃO IN VITRO DE EMBRIÕES BOVINOS COM TRANSPORTE DE OÓCITOS E DE EMBRIÕES POR LONGAS DISTÂNCIAS
}

\author{
Marcus Vinícius Galvão Loiola ${ }^{1}$, Marcos Chalhoub $^{2}$, AleXandra Soares Rodrigues ${ }^{1}$,

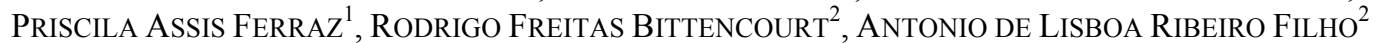 \\ ${ }^{1}$ Pós-graduandos da Universidade Federal da Bahia, Salvador, BA, Brasil. vinicius_mev@hotmail.com \\ ${ }^{2}$ Professores Doutores da Universidade Federal da Bahia, Salvador, BA, Brasil
}

RESUMO

\begin{abstract}
Objetivou-se avaliar a viabilidade de um programa de produção in vitro de embriões (PIVE) bovinos cuja maturação oocitária e cultivo embrionário ocorreram parcialmente durante o transporte, a fertilização (FIV) foi realizada com sêmen convencional ou sexado e os embriões foram transferidos com diferentes idades de desenvolvimento. Para tanto, oócitos aspirados no estado da Bahia foram transportados para um laboratório comercial em São Paulo, onde realizaram-se as etapas de produção in vitro, sendo a FIV realizada com espermatozoides oriundos de sêmen convencional (grupo CONV) ou sexado (grupo SEX). No dia seis de cultivo, os embriões produzidos foram remetidos à Bahia e
\end{abstract}

transferidos às receptoras com diferentes idades de desenvolvimento $(6,5 ; 7,0$ e 7,5 dias $)$. As fêmeas tiveram média de $46,18 \pm 32,7$ oócitos recuperados e $30,74 \pm 24,3$ oócitos viáveis por aspiração. As taxas de embriões produzidos e de gestação foram de $32,85 \%$ e $33,12 \%$, respectivamente. O tipo de sêmen não influenciou no número de oócitos clivados e nas taxas de gestação, entretanto, influenciou no número de embriões produzidos e a idade do embrião interferiu nas taxas de gestação. Os resultados encontrados demonstraram que o transporte de oócitos e de embriões foi eficiente em um programa de PIVE bovinos, podendo ser utilizado em larga escala.

PALAVRAS-CHAVE: Bos indicus; sêmen sexado; transferência de embriões.

\section{VALIDATION OF A PROGRAM PRODUCTION IN VITRO EMBRYO CATTLE WITH TRANSPORT OF OOCYTES AND EMBRYO FOR LONG DISTANCE}

\section{ABSTRACT}

This study aimed to evaluate the program of in vitro embryo production (IVEP) feasibility whose cattle oocyte maturation and embryo development occurred partly during the transport, fertilization (IVF) was performed with conventional or sexed semen and the embryos were transferred at different periods of development. Therefore, oocytes aspirated in the state of Bahia were transported to a commercial laboratory in São Paulo city, where the steps of in vitro production continued, the IVF was performed with sperm derived from conventional semen (CONV group) or sexed group (SEX group). On the sixth day of cultivation, the embryos produced were sent to Bahia and transferred to recipients at different periods of development (6.5, 7.0 and 7.5 days). The females had a mean of $46.18 \pm 32.7$ oocytes recovered, and $30.74 \pm 24.3$ viable oocytes by aspiration. The rates of embryos produced and pregnancy were $32.85 \%$ and $33.12 \%$, respectively. The type of semen did not affect neither the number of cleaved oocytes nor the pregnancy rates, however, it affected the number of embryos produced, and the age of the embryo interfered in the pregnancy rates. The results demonstrated that the transport of oocytes and embryos was effective in cattle IVEP program, thus, it can be used in large scale.

KEYWORDS: Bos indicus; sexed semen; transfer of embryos. 


\section{INTRODUÇÃO}

Nos últimos anos, as fêmeas bovinas têm sido alvo de numerosas pesquisas visando um melhor aproveitamento de seus gametas, principalmente aquelas consideradas geneticamente superiores (1). Segundo Bols et al (2), bezerras ao nascimento possuem mais de 100 mil oócitos em seus ovários, que, pelas vias naturais, podem dar origem a $0,01 \%$ de produtos viáveis, algo próximo de dez descendentes em toda sua vida reprodutiva. Uma forma de melhorar a exploração desses gametas é por meio das biotecnologias da reprodução, como a indução de múltipla ovulação e transferência de embriões (MOET) e a produção in vitro de embriões (PIVE) (3).

O Brasil passou por um crescimento significativo no segmento das biotecnologias na última década. Após um amplo e consolidado conhecimento sobre a obtenção de embriões in vivo, o país passou a dominar a aspiração folicular ou ovum pick up (OPU) e a PIVE, ocupando uma posição importante no mercado de embriões bovinos, sobretudo, por possuir o maior rebanho comercial do mundo e ser o principal exportador de carne bovina (4).

Atualmente, o método in vitro superou a MOET e se tornou a técnica de escolha na produção de embriões (4), especialmente, por ser mais utilizada em raças zebuínas, as quais fisiologicamente possuem uma maior população folicular, maior recuperação de oócitos por aspiração e, consequentemente, maior produção embrionária (5).

A PIVE é considerada uma ferramenta eficiente para a produção de animais de maior mérito genético, sendo um instrumento importante para exploração maximizada do potencial reprodutivo dos rebanhos, diminuindo o intervalo entre as gerações e acelerando o melhoramento genético animal (6). Vários fatores podem influenciar nos seus resultados: diferentes metodologias de maturação, cultivo, capacitação espermática, individualidade da doadora e do touro, entre outros (7).

O crescimento desta biotecnologia no Brasil permitiu sua aplicação em larga escala e a exportação desse modelo para vários países latinoamericanos e de outros continentes (8). Entretanto, a grande extensão territorial e a distância entre as propriedades onde ficam os animais e os laboratórios de PIVE, muitas vezes, têm limitado a expansão da produção in vitro comercial, principalmente pelas condições e pelo tempo gasto com o transporte dos oócitos e dos embriões (911).

Alternativas de transporte têm sido estudadas, um exemplo é o uso de incubadoras portáteis capazes de simular o ambiente do laboratório, permitindo, assim, tanto a maturação dos oócitos, quanto o cultivo dos embriões enquanto são transportados, o que possibilita a execução de todo o processo de produção in vitro sem nenhuma interrupção até o momento de transferência para as receptoras (12).

Entretanto, são poucos os estudos envolvendo essas temáticas e os resultados apresentados na literatura ainda são controversos $(9,12)$. O estudo de estratégias que possibilitem o transporte de oócitos e de embriões e o impacto que a utilização de doses de sêmen sexado, assim como a idade do embrião no momento da inovulação sobre os resultados desses procedimentos, possibilitará um melhor entendimento e elementos auxiliares ao emprego dessa tecnologia por técnicos e criadores.

Dessa forma, objetivou-se com este trabalho avaliar a viabilidade de um programa de PIVE bovinos da raça Nelore cuja maturação oocitária e cultivo embrionário ocorreram parcialmente durante o transporte, determinar o efeito da fertilização com sêmen sexado para fêmea sobre o número de oócitos clivados, a produção de embriões e a taxa de gestação e o efeito da idade do embrião no momento da inovulação sobre as taxas de gestação em um programa de PIVE com transporte de oócitos e embriões por longas distâncias.

\section{MATERIAL E MÉTODOS}

Foram utilizadas, como doadoras de oócitos, 123 fêmeas Bos taurus indicus da raça Nelore, selecionadas com base no mérito genético. Esses animais apresentavam escore de condição corporal médio de $3,80 \pm 0,5$ (escala de 1-5) (13), idade média de $6,00 \pm 3,1$ anos e tinham atividade ovariana regular (baseado em exame de palpação retal e ultrassonografia). Foram realizadas 274 sessões de OPU, com média de 2,23 $\pm 1,1$ procedimentos por animal, sendo que nenhum tratamento hormonal foi realizado antes das sessões. 
Novilhas e vacas mestiças (Bos taurus indicus $\mathrm{x}$ Bos taurus taurus) foram utilizadas como receptoras. Esses animais foram avaliados por exames ginecológicos e sanitários, receberam as vacinas obrigatórias e contra as principais doenças da esfera reprodutiva sendo mantidas a pasto com água e sal mineral à vontade.

O experimento ocorreu entre os anos de 2011 e 2012. Todos os animais que participaram (doadoras e receptoras) estavam em fazendas comerciais localizadas no estado da Bahia e a produção in vitro dos embriões ocorreu em um laboratório comercial no estado de São Paulo a uma distância aproximada de 2.000 quilômetros das fazendas onde estavam os animais.

As doadoras foram submetidas ao processo de aspiração folicular, realizado utilizando-se equipamento de ultrassonografia (ALOKA SSD 500, Aloka, Japão) com transdutor microconvexo de $5 \mathrm{MHz}$ conectado a uma guia de biópsia com agulha hipodérmica de 19G (Becton Dickinson, Curitiba, Brasil) e linha de aspiração (Corning, Acton, MA, EUA) em tubo de centrífuga de $50 \mathrm{~mL}$ aquecido $\left(37^{\circ} \mathrm{C}\right)$. A pressão de vácuo foi obtida com uma bomba de aspiração (BV004, WTA, Cravinhos, Brasil), ajustada entre 72 e $78 \mathrm{mmHg}$, permitindo um fluxo de $20 \mathrm{~mL}$ de meio/minuto.

Antes de cada procedimento foi realizada limpeza e assepsia da região perianal com água e álcool 70\%. Para evitar movimentos peristálticos e desconforto ao animal, foi feita anestesia epidural com $5 \mathrm{~mL}$ de Lidocaína a $2 \%$ (Anestésico L, Pearson, São Paulo, Brasil). Em seguida o transdutor foi inserido até o fundo de saco vaginal e, com o auxílio da manipulação retal, os ovários foram posicionados para uma boa visualização dos folículos na tela do ultrassom.

Os folículos visualizados foram posicionados no percurso da linha de punção e então aspirados pela agulha conectada ao sistema com pressão negativa acionada pela bomba de vácuo. $\mathrm{O}$ mesmo procedimento foi repetido em todos os folículos visualizados em ambos os ovários. O meio de colheita dos oócitos e de lavagem da agulha e do sistema era composto de DPBS (Dulbecco Mod. PBS, Vitrocell, Campinas, Brasil) acrescido de 5,0 UI/mL de heparina sódica (Liquemine, Roche, Rio de Janeiro, Brasil).

O material aspirado foi transferido para um filtro de colheita de embriões (Vitrocell, Campinas, Brasil) e lavado com a mesma solução usada na aspiração. O sedimento restante no filtro foi transferido para placas de Petri (TPP,
Vitrocell, Campinas, Brasil) e observado em microscópio estereoscópio (SZM 1000, Nikon, Melville, EUA), onde se procedeu a busca e classificação dos oócitos de acordo com a sua morfologia (número de camadas e o grau de expansão das células do cumulus e o aspecto do citoplasma quanto à cor, homogeneidade e integridade) em grau I, II, III e IV, segundo Seneda et al. (14).

Os oócitos considerados viáveis (grau I, II e III) foram lavados em solução de TCM 199 Hepes (Gibco Life Techonologies, Grand Island, EUA), suplementada com 10\% de SFB (Vitrocell, Campinas, Brasil), $50 \mu \mathrm{g}$ de gentamicina

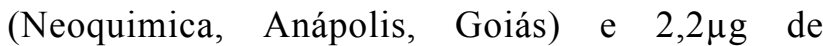
piruvato (Sigma-Aldrich, St. Louis, EUA). Após essa etapa, iniciou-se a maturação in vitro, quando foram acondicionados em criotubos (Uniscience, São Paulo, Brasil) com $400 \mu \mathrm{L}$ de meio de maturação constituído de TCM-199 bicarbonato (Gibco Life Techonologies, Grand Island, EUA); suplementado com 10\% de SFB (Vitrocell, Campinas, Brasil); 50UI de $\mathrm{hCG} / \mathrm{mL}$ (Biodux, Campinas, Brasil); $0,5 \mu \mathrm{g} / \mathrm{mL}$ de FSH (Vetrepharm, Belleville, Canada); $1 \mu \mathrm{g} / \mathrm{mL}$ de estradiol (Sigma-Aldrich, St. Louis, EUA); $2,2 \mu \mathrm{g} / \mathrm{mL}$ de piruvato (Sigma-Aldrich, St. Louis, EUA) e $70 \mu \mathrm{g} / \mathrm{mL}$ de amicacina (Instituto Biochimico, Rio de Janeiro, Brasil). Este meio foi recoberto com $300 \mu \mathrm{L}$ de óleo mineral e, por fim, o ambiente interno do criotubo (Uniscience, São Paulo, Brasil) foi gaseificado com uma mistura de $5 \%$ de $\mathrm{CO}_{2}$ e os oócitos foram submetidos ao transporte em incubadora portátil (Minitube, Tiefenbach, Alemanha) a uma temperatura de $38,5{ }^{\circ} \mathrm{C}$.

Os oócitos foram transportados de carro até o aeroporto mais próximo e então remetidos de avião para o laboratório no estado de São Paulo. O transporte durou entre 18 e 24 horas e, após chegar ao laboratório, já em processo de maturação, foram transferidos para placas de Petri (TPP, Vitrocell, Campinas, Brasil), em microgotas de $100 \mu \mathrm{L}$ de meio de maturação semelhante ao usado no transporte até completarem o período de 24 horas de maturação, a $38,5{ }^{\circ} \mathrm{C}$ em atmosfera de $5 \%$ de $\mathrm{CO}_{2}$ em ar.

Percorrido o tempo de maturação, os oócitos foram transferidos para microgotas de $100 \mu \mathrm{L}$ de meio de fecundação Tyrode modificado (TALP) suplementado com $10 \mu \mathrm{g} / \mathrm{mL}$ de heparina e $160 \mu \mathrm{L} / \mathrm{mL}$ de solução de epinefrina (SigmaAldrich, St. Louis, EUA). Para a fertilização, foi 
utilizado sêmen criopreservado comercializado de 12 touros diferentes da raça Nelore. A depender do acasalamento sugerido pelos proprietários das doadoras, os oócitos maturados foram direcionados para fertilização com espermatozoides provenientes de sêmen convencional (grupo 1 - CONV) ou sêmen sexado para fêmea (grupo 2 - SEX). Em ambos os grupos, o sêmen foi separado em gradiente de Percoll 90 e $45 \%$ (4mL), submetido a uma força de centrifugação de $200 \mathrm{~g}$ durante 30 minutos. O sobrenadante foi descartado e o pellet recuperado foi avaliado e ajustado de modo a obter a concentração final de $2 \times 10^{6}$ espermatozoides viáveis $/ \mathrm{mL}$. Posteriormente, foram incubados por 18 horas a $39^{\circ} \mathrm{C}$ em atmosfera de $5 \%$ em ar, para a fecundação.

Após o tempo de fecundação, as estruturas foram lavadas três vezes e transferidas para microgotas de $100 \mu \mathrm{L}$ de meio de cultivo (Synthetic Oviduct Fluid) SOF modificado, contendo 8 $\mathrm{mg} / \mathrm{mL}$ de BSA e $1 \mathrm{mM}$ de glutamina (SigmaAldrich, St. Louis, EUA) recobertas com óleo mineral. O meio de cultivo foi renovado em cada microgota no terceiro e no quinto dia (feeding), permanecendo nestas por um período de seis dias, sendo que no segundo dia de cultivo foi avaliado o número de oócitos clivados. Os embriões produzidos foram transportados de volta à Bahia, fazendo o mesmo percurso dos oócitos, para serem transferidos às receptoras.

$\mathrm{O}$ transporte dos embriões ocorreu em condições semelhantes às dos oócitos, entretanto, em meio de cultivo embrionário. Devido à logística dos percursos, foram feitos cálculos do tempo gasto com o transporte (em média 18 a 24 horas) de forma que os embriões retornavam no dia seis (D0 - dia da FIV) e foram inovulados entre os dias seis e sete. Ao fim do transporte, os embriões (blastocistos e blastocistos expandidos) foram novamente avaliados e envasados em palhetas de $0,25 \mathrm{~mL}$ para serem transferidos.

Devido à distância entre o laboratório e as fazendas onde se encontravam as receptoras, os embriões foram transferidos com diferentes idades de desenvolvimento. Sendo assim, em um percentual dos embriões produzidos $(n=893)$, foi avaliada a influência da inovulação de embriões com diferentes idades (D0 - dia da FIV), perfazendo três grupos experimentais: embriões inovulados com 6,5 dias de idade, TE-6,5D $(n=457)$; embriões inovulados com 7,0 dias de idade, TE-7,0D $\quad(n=322)$; inovulações com embriões de 7,5 dias de idade, TE-7,5D (n=114).

As receptoras utilizadas foram submetidas a um protocolo de transferência de embrião em tempo fixo, sendo que, no dia 0 , receberam um dispositivo intravaginal de progesterona $\left(\mathrm{DIB}^{\circledR}\right.$, MSD Saúde animal, São Paulo, Brasil) associado a $2 \mathrm{mg}$ de Benzoato de Estradiol i.m. (Gonadiol ${ }^{\circledR}$, MSD Saúde animal, São Paulo, Brasil). No dia 8, os dispositivos foram retirados e foram aplicados 300UI de Gonadotrofina Coriônica Equina i.m. (eCG, Novormon ${ }^{\circledR}$, MSD Saúde animal, São

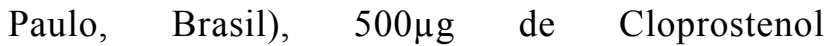
i.m.(Ciosin ${ }^{\circledR}$, MSD Saúde animal, São Paulo, Brasil) e $1 \mathrm{mg}$ de Cipionato de Estradiol i.m. $\left(\mathrm{ECP}^{\circledR}\right.$, Pfizer, Guarulhos, Brasil). O dia 10 foi considerado como o dia do estro e os embriões foram transferidos no dia 17 do protocolo. Anteriormente a cada inovulação, os mesmos procedimentos referentes à limpeza, assepsia e anestesia realizados nas doadoras foram conferidos às receptoras. Também foram realizados exames dos ovários por ultrassonografia (ALOKA SSD 500, Aloka, Japão) para confirmar a presença e o tamanho do corpo lúteo (CL), sendo que apenas receptoras com $\mathrm{CL}$ maior que $13 \mathrm{~mm}$ receberam embrião.

O diagnóstico de gestação foi realizado por ultrassonografia transretal, 30 dias após a data da FIV e repetido com 60 dias, utilizando transdutor linear de 5,0MHz (ALOKA SSD 500, Aloka, Japão). A presença de uma vesícula embrionária com viabilidade confirmada (batimento cardíaco) foi considerada diagnóstico de gestação positivo. A taxa de gestação foi calculada dividindo o total de receptoras gestantes pelo total de vacas que receberam embrião.

Os dados foram processados usando-se o Statistical Package for Social Science (SPSS, versão 19). Para tanto, realizou-se a seguinte sequência de análises: 1- a média e o desvio padrão das características de interesse ao estudo foram obtidas por meio da análise descritiva; 2 - as diferenças entre os números de oócitos clivados e os números de embriões produzidos entre os grupos CONV e SEX foram comparados utilizando-se o teste T de Student; 3- as taxas de gestação entre os grupos CONV e SEX e entre os grupos com embriões de diferentes idades (TE6,5D; TE-7,0D; TE-7,5D) foram comparadas empregando-se um estudo de dispersão de frequências utilizando o teste de Qui-quadrado $\left(\chi^{2}\right)$. Os testes foram realizados considerando um nível de significância de 0,05 . 


\section{RESULTADOS E DISCUSSÃO}

Um total de 12.653 oócitos foi recuperado em 274 sessões de aspiração. Em média, $46,18 \pm 32,7$ oócitos foram obtidos por OPU e $8.423(66,56 \%)$ foram considerados viáveis, gerando uma média de $30,74 \pm 24,3$ oócitos viáveis por procedimento (Tabela 1). Esses resultados divergem dos obtidos por Viana et al. (4), que analisaram os dados de quatro consolidadas empresas brasileiras produtoras de embriões bovinos in vitro e encontraram média de 19,90 oócitos por OPU, variando entre 15,20 e 24,40. Entretanto, trabalhando apenas com animais da raça Nelore (Bos taurus indicus), Pontes et al. (15) analisaram o desempenho de 317 doadoras durante 656 sessões de aspiração folicular em um centro comercial de produção in vitro de embriões e encontraram média de $30,84 \pm 0,88$ oócitos recuperados por aspiração, sendo $23,35 \pm 0,72$ considerados viáveis. Da mesma forma, Rubin et al. (16) encontraram média de 25,14 oócitos viáveis por OPU em fêmeas Nelore.

Tabela 1. Taxas, médias e desvio padrão (dp) de oócitos totais recuperados (OOTO), oócitos viáveis recuperados (OOVI), embriões produzidos (EMB) e de gestação (GES) obtidas a partir de doadoras Nelore submetidas a aspiração folicular guiada por ultrassonografia (OPU) e a Produção in vitro de embriões (PIVE)

\begin{tabular}{lcc}
\hline Variáveis & Taxa $(\%)$ & Média $\pm(d p) /$ Sessão OPU-PIVE \\
\hline \multirow{2}{*}{ OOTO } & ---- & $46,18 \pm 32,7$ \\
& & $(12.653 / 274)$ \\
OOVI & 66,56 & $30,74 \pm 24,3$ \\
& $(8.423 / 12.653)$ & $(8.423 / 274)$ \\
EMB & 32,85 & $10,09 \pm 6,2$ \\
& $(2.767 / 8.423)$ & $(2.767 / 274)$ \\
GES & 33,12 & $2,71 \pm 1,2$ \\
& $(745 / 2.249)$ & $(745 / 274)$ \\
\hline
\end{tabular}

A disponibilidade de oócitos é considerada por vários autores como uma das características que mais influencia a produção de embriões in vitro. Essa característica apresenta grande variabilidade individual e depende de diversas variáveis fisiológicas ou patológicas, como idade, padrão nutricional, estação do ano, temperatura e fatores genéticos (1). A forma como cada procedimento de aspiração folicular é realizado pela equipe técnica também influencia na recuperação oocitária: a quantidade de punções, o tipo de bomba de vácuo, a pressão utilizada, o tipo de agulha, a eficiência do técnico e modificações na classificação dos oócitos são fatores extrínsecos que podem influenciar nos resultados finais da PIVE, justificando as divergências encontradas na literatura e os resultados aqui expostos $(17,16,18)$.

A taxa de embriões produzidos, a partir de oócitos que foram submetidos à maturação durante $\mathrm{o}$ transporte em incubadora portátil com duração de 18 à 24 horas foi de $32,85 \%$ (Tabela 1), tendo uma média de 10,09 $\pm 6,2$ embriões produzidos por aspiração. Esses resultados corroboram os encontrados por Alves et al. (9), os quais avaliaram a influência do transporte de oócitos na produção in vitro de embriões bovinos e conseguiram uma taxa de embriões produzidos de 33,6\%; entretanto, neste estudo o transporte teve duração de seis horas e ocorreu em tubos de poliestireno de $5 \mathrm{~mL}$ a $30{ }^{\circ} \mathrm{C}$. Esses autores não encontraram efeito do transporte de oócitos sobre o desenvolvimento embrionário in vitro, sugerindo-o como uma alternativa para melhorar o emprego da OPU/PIVE.

Contudo, Leivas et al. (19), ao trabalharem com transporte de oócitos bovinos em meio de maturação associado a um tampão orgânico Hepes, sem controle de atmosfera gasosa a $39{ }^{\circ} \mathrm{C}$, verificaram uma redução significativa do desenvolvimento embrionário quando a duração do transporte prolongou-se por até 18 horas. Esses resultados foram atribuídos a uma possível incapacidade do tampão Hepes em manter o $\mathrm{pH}$ por até 18 horas, o que pode ter determinado prejuízos no desenvolvimento posterior dos embriões.

O modelo de transporte de oócitos usado neste experimento foi eficiente mesmo quando o transporte foi prolongado por mais de 18 horas, a produção de embriões encontrada neste estudo apresentou-se em acordo com a média nacional, relatada por Brum et al. (20), de $35 \%$ de blastocisto 
no dia sete de cultivo. Esses resultados podem ser justificados pela menor variação do $\mathrm{pH}$ do meio através do controle da atmosfera gasosa com a gaseificação prévia dos criotubos, mantendo $5 \%$ de $\mathrm{CO}_{2}$ em ar e o controle eficiente da temperatura através da incubadora portátil, simulando as condições encontradas no laboratório durante a maturação in vitro.

A quantidade de embriões produzidos, em alguns momentos, superou a quantidade de receptoras disponíveis para transferência, dessa forma, em determinadas situações ao longo do experimento parte dos embriões produzidos foram descartados. Então, do total de 2.767 embriões, 2.249 foram transferidos (Tabela 1). A taxa de gestação foi de $33,12 \%$, correspondendo a uma média de 2,71 $\pm 1,2$ gestações por OPU. De forma semelhante, Pontes et al. (5) submeteram embriões bovinos produzidos in vitro ao transporte por longas distâncias nas mesmas condições do presente estudo e encontraram taxas de gestação total de $39 \%$. Porém, a média de gestações por procedimento foi inferior a aqui apresentada, provavelmente pelo menor número de oócitos recuperados por doadora.

As taxas de gestação encontrada neste estudo são similares às citadas por Viana et al. (21) a partir de comunicações pessoais de renomadas empresas de PIVE bovinos do Brasil (38,5\%, com média de 2,7 prenhez/OPU/doadora), demostrando que as condições utilizadas no transporte foram eficientes e obterão resultados semelhantes a programas de PIVE realizados sem o transporte prévio de oócitos e embriões, possibilitando o uso dessa biotecnologia em larga escala por viabilizar tanto o envio de oócitos quanto de embriões de fazendas distantes dos laboratórios que oferecem esses serviços.

A influência do sêmen sexado na produção de embriões bovinos in vitro também foi estudada (Tabela 2). O número de oócitos clivados não diferiu $(\mathrm{P}=0,49)$ quanto ao uso de sêmen convencional (CONV $=61,49 \%$ ) ou sêmen sexado (SEX $=63,43 \%$ ). A taxa de gestação com os diferentes tipos de sêmen também foi similar $\quad(\mathrm{CONV}=33,37 \% \quad$ e $\mathrm{SEX}=31,93 \%, \mathrm{P}=0,586)$; contudo, o número de embriões produzidos com sêmen sexado para fêmea ( $\mathrm{SEX}=47,76 \%)$ foi superior $(\mathrm{P}=0,038)$ ao número de embriões com sêmen convencional $(\mathrm{CONV}=47,76 \%)$. Diferindo desses achados, Blondin et al. (22) encontraram maior número de clivagem e menor produção de blastocisto quando o sêmen sexado foi utilizado na PIVE em comparação ao sêmen convencional. Na mesma pesquisa também foi observado um comprometimento da qualidade do sêmen devido ao processo de sexagem, o que pode explicar a redução nas taxas de blastocisto.

Vários estudos têm mostrado que o processo de sexagem envolve uma série de passos que podem causar alterações na funcionalidade da membrana, características de motilidade e morfologia dos espermatozoides, consequentemente, diminuição na fertilidade quando o sêmen sexado é utilizado em relação ao sêmen convencional (23). Entretanto, neste estudo, houve maior produção de embriões quando o sêmen sexado foi utilizado.

Tabela 2. Comparação do número de oócitos clivados (OOCLIV), de embriões produzidos (EMBPR) e da taxa de gestação (TXGES) em um programa de Produção in vitro de Embriões (PIVE) utilizando sêmen convencional (CONV) ou sêmen sexado (SEX) com transporte de oócitos e de embriões por longas distâncias

\begin{tabular}{lccc}
\hline \multirow{2}{*}{ Variáveis } & \multicolumn{2}{c}{ Tipo de sêmen usado (convencional ou sexado) } & \multirow{2}{*}{ P-Valor } \\
\cline { 2 - 3 } & CONV & SEX & 0,490 \\
\hline OOCLIV (\%)* & 61,49 & 63,43 & 0,038 \\
EMBPR (\%)* & $38,11 \mathrm{a}$ & $47,76 \mathrm{~b}$ & 0,586 \\
TXGES (\%)** & 33,37 & 31,93 & 0,586 \\
\hline
\end{tabular}

Valores seguidos de letras distintas na mesma linha diferem entre si $(\mathrm{P}<0,05)$.

Divergindo desses achados, Carvalho et al. (24) não encontraram diferença para fertilização, clivagem dos oócitos e taxa de blastocisto no dia oito, quando utilizaram sêmen convencional, sexado para macho ou sexado para fêmea. Da mesma forma, $\mathrm{Xu}$ et al. (25) observaram taxas de gestação com o uso de sêmen sexado na PIVE em bovinos semelhantes ao sêmen convencional.

Possivelmente, variações nos protocolos de separação dos espermatozoides antes da fertilização (tipos e volumes de gradientes, força e duração da centrifugação), bem como variações nas concentrações de heparina (utilizada para capacitação espermática in vitro) e no tamanho da 
gota de fertilização, possam ser responsáveis pela divergência em relação aos resultados encontrados referentes ao uso de sêmen sexado na produção in vitro $(22,24,26)$.

Além disso, o processo de sexagem torna mais evidente a variação individual entre touros. Alguns animais apresentam maior comprometimento da qualidade seminal após este processo e necessitam de concentrações diferentes de heparina na capacitação para produzirem maiores quantidades de embriões, podendo justificar os resultados encontrados (22). Uma forma de maximizar a eficiência do sêmen sexado na PIVE seria o monitoramento, por parte das empresas comerciais, dos resultados individuais de cada touro e a classificação dos animais que apresentam maior fertilidade para espermatozoides sexados (24), porém, neste experimento, foram utilizadas doses de sêmen comercial de 12 diferentes touros, não sendo comparados quanto aos parâmetros espermáticos e suas possíveis variações.

Por consequência do transporte dos embriões até as fazendas de receptoras, as transferências ocorriam com embriões em diferentes idades de desenvolvimento e essa característica foi analisada em relação à sua influência nas taxas de gestação (Tabela 3). As taxas de gestação entre os embriões transferidos com 6,5 e 7,0 dias de idade não diferiram (TE-6,5D: 36,10\% vs. TE-7,0D: 40,99\%, $\mathrm{P}=0,167$ ), assim como entre os embriões de 7,0 e 7,5 dias (TE-7,0D: 40,99\% vs. TE-7,5D: $50,00 \%$, $\mathrm{P}=0,095)$; no entanto, houve diferença entre as taxas de gestação dos embriões inovulados com 6,5 e 7,5 dias de idade de desenvolvimento (TE-6,5D: 36,10\% vs. TE-7,0D: 50,00\%, $\mathrm{P}=0,006$ ).

Tabela 3. Comparação entre as taxas de gestação de receptoras que tiveram os embriões transportados por longas distâncias e inovulados com 6,5 dias (TE-6,5D), 7,0 dias (TE-7,0D) e 7,5 dias de idade (TE-7,5D)

\begin{tabular}{|c|c|c|}
\hline Variáveis & Embriões inovulados & $\begin{array}{c}\text { Taxa de gestação } \\
(\%)\end{array}$ \\
\hline TE-6,5D & 457 & $\begin{array}{c}36,10 \mathrm{a} \\
(165 / 457)\end{array}$ \\
\hline TE-7,0D & 322 & $\begin{array}{c}40,99 \mathrm{ab} \\
(132 / 322)\end{array}$ \\
\hline TE-7,5D & 114 & $\begin{array}{c}50,00 \mathrm{~b} \\
(57 / 114) \\
\end{array}$ \\
\hline TOTAL & 893 & $\begin{array}{c}39,64 \\
(354 / 893) \\
\end{array}$ \\
\hline
\end{tabular}

Valores seguidos de letras distintas na mesma coluna diferem entre si $(\mathrm{P}<0,05)$ pelo teste do Qui-Quadrado

Os embriões que foram inovulados com a idade mais avançada apresentaram melhores taxas de gestação. Esses embriões permaneceram mais tempo em meio de cultivo embrionário e foram transferidos no turno em que tinha menor estresse térmico. Além disso, eram mais velhos no momento da transferência, quando comparados aos dos outros grupos, possuindo, assim, maior capacidade de produção de células trofoblásticas e, consequentemente, maior produção de interferon-tau, o que pode ter contribuído para um maior reconhecimento materno e melhores taxas de gestação (27).

Acredita-se que o conjunto de fatores descritos acima pode ter contribuído para uma melhor interação entre $\mathrm{o}$ ambiente uterino das receptoras e o embrião, diminuindo as perdas embrionárias e melhorando as taxas de gestação deste grupo; entretanto, mais estudos são necessários envolvendo os fatores que podem influenciar no momento da inovulação em relação às taxas de gestação em programas de PIVE.

\section{CONCLUSÕES}

O transporte de oócitos e de embriões nas condições realizadas neste experimento mostrou-se eficiente, obtendo-se resultados satisfatórios de produção de embriões e taxas de gestação, viabilizando, assim, programas de PIVE em bovinos quando doadoras e receptoras encontram-se distantes do laboratório de produção de embriões.

A utilização de sêmen sexado para fêmea não interferiu no número de oócitos clivados e nas taxas de gestação, quando comparado com o sêmen convencional, mas favoreceu a produção de embriões, mostrando ser uma excelente ferramenta associada à PIVE para a produção de animais de alto mérito genético com a pré-escolha do sexo, estando à disposição dos técnicos e criadores.

A idade do embrião no momento da inovulação é um parâmetro importante em 
programas de PIVE bovinos com transporte por longas distâncias, sendo que as melhores taxas de gestação foram obtidas com embriões mais velhos, necessitando-se de mais estudos que possam justificar os motivos dessa melhora.

\section{REFERÊNCIAS}

1. Seneda MM, Esper CR, Garcia JM, Andrade ER Aspectos técnicos e biológicos da obtenção de oócitos bovinos: revisão de literatura [Technical and biological aspects of obtaining bovine oocytes: a literature review]. Semina: Ciências Agrárias. 2002;23(1):101-10. Portuguese.

2. Bols PE, Ysebaert MT, Van Soom A, Kruif A. Effects of needle tip bevel and aspiration procedure on the morphology and developmental capacity bovine compact cumulus oocyte complexes. Theriogenology. 1997;47:1221-36.

3. Merton JS, Roos AP, Mullaart E, Ruigh L, Kaal L, Vos PL, et al. Factors affecting oocyte quality and quantity in commercial application of embryo technologies in the cattle breeding industry. Theriogenology. 2003;59:651-74.

4. Viana JH, Siqueira LG, Palhao MP, Camargo LS. Features and perspectives of the Brazilian in vitro embryo industry. Animal Reproduction. 2012;9(1):12-18.

5. Pontes JH, Silva KC, Basso AC, Rigo AG, Ferreira $\mathrm{CR}$, Santos GM, et al. Large-scale in vitro embryo production and pregnancy rates from Bos taurus, Bos indicus, and indicus-taurus dairy cows using sexed sperm. Theriogenology. 2010;74:1349-55.

6. Varago FC, Mendonça LF, Lagares MA. Produção in vitro de embriões bovinos: estado da arte e perspectiva de uma técnica em constante evolução [In vitro production of bovine embryos: state of the art and perspective of a constantly evolving technical]. Revista Brasileira de Reprodução Animal. 2008;32(2):100-09. Portuguese.

7. Camargo LS, Viana JH, Sá WF, Ferreira AM, Ramos AA, Vale Filho VR. Factors influencing in vitro embryo production. Animal Reproduction. 2006;3(1):19-28.

8. Bols PE, Jorssen EP, Goovaerts IG, Langbeen A, Leroy JL. High throughput non-invasive oocyte quality assessment: the search continues. Animal Reproduction. 2012;9(3):420-25.

9. Alves DF, Rauber LP, Rubin FB, Bernardi ML, Dezen $\mathrm{D}$, Silva CA, et al. Desenvolvimento embrionário in vitro de oócitos bovinos mantidos em líquido folicular ou TCM-hepes [Embryo development in vitro of bovine oocytes maintained in follicular fluid or TCM-Hepes]. Brazilian Journal of Veterinary Research and Animal Science. 2003;40:279-86. Portuguese.

10. Marinho LS, Untura RM, Morotti F, Moino LL, Rigo AG, Sanches BV, et al. Large-scale programs for recipientes of in vitro-produced embryos. Animal
Reproduction. 2012;9(3):323-28.

11. Tessmann JV, Mozzaquatro DF, Rauber LP, Santos MV, Chequim RM, Bernardis ML, et al. Transportematuração de oócitos bovinos em palhetas [Trans-maturity bovine oocytes in straws]. Acta Scientiae Veterinariae. 2004;32(3):177-84. Portuguese.

12. Max MC, Santos GM, Melo-Sterza FA, Silva-Santos $\mathrm{KC}$, Morotti F, Basso AC, et al. In vitro embryo production in sheep: Pregnancy after long periods of oocyte and embryo transport. Small Ruminant Research. 2012;105:286-89.

13. Houghton PL, Lemenager RP, Moss GE, Hendrix KS. Prediction of postpartum beef cow body composition using weight to height ratio and visual body condition score. Jornal Animal Science. 1990;68:1428-37.

14. Seneda MM, Esper CR, Garcia JM, Oliveira JA, Vantini R. Relationship between follicle size and ultrasound-guided transvaginal oocyte recovery. Animal Reproduction Science. 2001;67:37-43.

15. Pontes JH, Sterza FA, Basso AC, Ferreira CR, Sanches BV, Rubin KC, et al. Ovum pick up, in vitro embryo production, and pregnancy rates from a largescale commercial program using Nelore cattle (Bos indicus) donors. Theriogenology. 2011;75:1640-46.

16. Rubin KC, Rigo AG, Schroeder RV, Silva RC, Marques MO, Seneda MM. Avaliação de uma bomba de infusão continua como geradora de vácuo para obtenção in vivo de oócitos bovinos [Evaluation of an infusion pump continues as generating vacuum to obtain in vivo bovine oocytes]. Acta Scientiae Veterinariae. 2004;32:121

17. Pontes JH, Nonato-Junior I, Sanches BV, Ereno-Junior JC, Uvo S, Barreiros RR, et al. Comparison of embryo yield and pregnancy rate between in vivo and in vitro methods in the same Nelore (Bos indicus) donor cows. Theriogenology. 2009;71:690-700.

18. Viana JH, Bols PE. Variações biológicas associadas a recuperação de complexos cumulus-oócitos por aspiração folicular [Biological variations associated with recovery of cumulus-oocyte complexes by follicular aspiration]. Acta Scientiae Veterinariae. 2005;33:1-4. Portuguese.

19. Leivas FG, Brun DS, Mezzalira A, Pilla LF, Bernardi ML, Rubin MI, et al. Transporte de oócitos bovinos em meio de maturação sem controle de atmosfera gasosa [Transport of bovine oocytes in maturation medium without controlled gaseous atmosphere]. Ciência Rural. 2004;34(1):219-24. Portuguese.

20. Brum DS, Leivas FG, Bernardi ML, Rauber LP, Mezzalira A, Brass KE, et al. Cultivo individual de blastocistos bovinos produzidos in vitro [Individual cultivation of bovine blastocysts produced in vitro]. Brazilian Journal of Veterinary Research and Animal Science. 2002;39(2):87-92. Portuguese.

21. Viana JH, Siqueira LG, Palhao MP, Camargo LS. Use of in vitro Fertilization Technique in the Last Decade and 
its Effect on Brazilian Embryo Industry and Animal 30.

Production. Acta Scientiae Veterinariae. 2010;38:661-74.

25. Xu J, Guo ZS, Nedambale TL, Zhang J, Schenk J.

22. Blondin P, Beaulieu M, Founier V, Morin N, Developmental potential of vitrified Holstein cattle Crawford L, Madan P, et al. Analysis of bovine sexed embryos fertilized in vitro with sex-sorted sperm. Journal sperm for IVF from sorting to the embryo. Dairy Science. 2006;89:2510-18.

Theriogenology. 2009; 71:30-38.

26. Wheeler MB, Rutledge JJ, Fischer-Brown A, Vanetten 23. Arruda RP, Celeghini EC, Alonso MA, Carvalho HF, Lemes KM, Silva DF, et al. Aspects related to the technique and the utilization of sexed semen in vivo and in vitro. Animal Reproduction. 2012;9(3):345-53.

24. Carvalho JO, Sartori R, Machado GM, Mourão GB, Dode MA. Quality assessment of bovine cryopreserved sperm after sexing by flow cytometry and their use in vitro embryo production. Theriogenology. 2010;74:1521-

T, Malusky S, Beebe DJ. Application of sexed semen technology to in vitro embryo production in cattle. Theriogenology. 2006;65:219-27.

27. Mann GE, Lamming GE. Relationship between maternal endocrine environment, early embryo development and inhibition of the luteolytic mechanism in cows. 2001;121:175-80. 